\title{
Abrindo os armários do acesso e da qualidade: uma revisão integrativa sobre assistência à saúde das populações LGBTT
}

\author{
Opening the closets of access and quality: an integrative review \\ on the health of LGBTT populations
}

Breno de Oliveira Ferreira (https://orcid.org/0000-0002-0979-3911) ${ }^{1}$

Cláudia Bonan (https://orcid.org/0000-0001-8695-6828) ${ }^{1}$

${ }^{1}$ Instituto Fernandes Figueira, Fiocruz. Av. Rui Barbosa 716, Flamengo. 22250-020 Rio de Janeiro RJ Brasil.

breno.oli@hotmail.com

\begin{abstract}
The research aimed to study the situations that condition access and quality of health care to lesbians, gays, bisexuals, transvestites and transsexuals (LGBTT) in health services from an integrative review of national and international literature, whose sample of 41 papers was selected in PubMed, Lilacs and SciELO databases from 2007 to 2018. Access and health issues of LGBTT people were discussed in three dimensions: relational, concerning intersubjective relationships among users and professionals; organizational, concerning the organization of services and work processes; and contextual, which covers the effect of vulnerable situations enmeshed with social determinants on the conditions of satisfaction of health needs. The related data showed that LGBTT populations are the target of prejudice, violence, and discrimination, which, added to different social indicators, engender a context of vulnerabilities in access and healthcare. It is necessary to transform health institutions' practices and social relationships. Otherwise, there is a risk of increasingly warding off those populations from health services.
\end{abstract}

Key words Sexual and gender minorities, Health services accessibility, Health care, Quality health care, Integrative review
Resumo A pesquisa buscou estudar as situações que condicionam o acesso e a qualidade da assistência à saúde de lésbicas, gays, bissexuais, travestis e transexuais (LGBTT) nos serviços de saúde, a partir de uma revisão integrativa da literatura nacional e internacional, cuja amostra de 41 artigos foi selecionada nas bases PubMed, Lilacs e SciELO, no período entre 2007 e 2018. O acesso e a assistência à saúde das populações LGBTT foram discutidos em três dimensões: relacional, que diz respeito às relações intersubjetivas entre usuários e profissionais; organizacional, que se refere aos modos de organizar os serviços e os processos de trabalho; e contextual, que engloba como situações de vulnerabilidades imbricadas com os determinantes sociais afetam as condições de satisfação das necessidades de saúde. Os dados mostraram que as populações LGBTT são alvo de preconceitos, violências e discriminações, que, somados a diferentes marcadores sociais, engendram um contexto de vulnerabilidades no acesso e na assistência à saúde. É necessário transformar as práticas e as relações sociais que se dão no interior das instituições de saúde, do contrário, corre-se o risco de afastar cada vez mais parte dessas populações dos serviços de saúde.

Palavras-chave Minorias sexuais e de gênero, Acesso aos serviços de saúde, Assistência à saúde, Qualidade da assistência à saúde, Revisão integrativa 


\section{Introdução}

O acesso aos serviços de saúde está relacionado à capacidade de um grupo para buscar e obter assistência à saúde, e as condições de acessibilidade comportam dimensões políticas, econômicas, técnicas e simbólicas ${ }^{1}$. Já a qualidade dos serviços de saúde costuma ser avaliada normativamente, tomando como marco teórico sete pilares: eficácia, efetividade, eficiência, otimização, aceitabilidade, legitimidade e equidade ${ }^{2}$. Contudo, a ideia de qualidade também está relacionada à própria subjetividade dos processos de saúde ${ }^{3}$.

Nesse diapasão, as desigualdades de acesso e a baixa qualidade da assistência à saúde são problemas apontados em diferentes sistemas de saúde pelo mundo, e afetam especialmente grupos minoritários, como populações de Lésbicas, Gays, Bissexuais, Travestis e Transexuais (LGBTT) ${ }^{4,5}$. O imaginário social que institui padrões sexuais binários e promove os alinhamentos "mulhervagina-maternidade-procriação-heterossexualidade" e "homem-pênis-racionalidade-paternidade-heterossexualidade"6 constrói um não lugar ou um lugar des-locado para aqueles que não se adequam às suas normas. Então, onde e como seriam acolhidos em suas diferentes necessidades de saúde? No armário?

A metáfora do armário encena como os serviços de saúde têm sido palco de negações e ocultações das sociabilidades das populações LGBTT, mas nessa discussão, destaca-se que a própria literatura científica sobre essa temática tem sido pouco visibilizada, por isso a importância de sublinhá-la como um grande armário ampliado. Trazer esse debate para fora do armário é também promover reflexões, incursões e criticidades no campo do conhecimento.

A orientação sexual e a identidade de gênero são fatores que devem ser considerados nos debates sobre determinação social em saúde. Os sujeitos que escapam ao binarismo sexual são expostos a inúmeras situações de vulnerabilidade, não apenas no que concerne aos cuidados em saúde, mas também a emprego, renda, estudos, segurança, dentre outras ${ }^{4}$.

O mundo da dissidência da norma heterossexual é muito vasto. As letras L, G, B, T e T percebidas apenas como um acrônimo podem levar à ilusão da homogeneidade. Cada uma dessas letras reúne, sob si mesma, um conjunto amplo e diversificado de experiências, segundo situações e marcadores interseccionais. Entretanto, as experiências dessas coletividades são atravessadas por algumas linhas de força em comum, estando su- jeitas aos estigmas, preconceitos e violências que se expressam também no acesso e na assistência à saúde $^{4-6}$. Tudo isso tem apontado para um longo caminho de disputas políticas, conflitos e negociações no campo dos direitos fundamentais e da cidadania ${ }^{7}$. Na modernidade, leis, normas, políticas, protocolos, entre outros, são instrumentos relevantes na promulgação desses direitos ${ }^{8}$.

Neste artigo, por meio de uma revisão integrativa da literatura nacional e internacional, são discutidos condicionantes e desafios ao acesso e à qualidade da assistência à saúde de lésbicas, gays, bissexuais, travestis e transexuais nos serviços de saúde.

\section{Percurso metodológico}

Trata-se de uma revisão integrativa da literatura nacional e internacional, método de pesquisa que tem por objetivo reunir, sintetizar e avaliar criticamente resultados de estudos anteriores sobre um determinado tema/problema, permitindo a construção de um panorama sobre o estado atual do conhecimento e a identificação de lacunas e direções para futuras pesquisas ${ }^{9}$. A questão a ser discutida neste estudo são as situações que condicionam o acesso e a qualidade da assistência à saúde de lésbicas, gays, bissexuais, travestis e transexuais nos serviços de saúde.

O levantamento das publicações científicas foi realizado em janeiro de 2019 nas seguintes bases de dados: USA National Library of Medicine (PubMed), Literatura Latino-Americana e do Caribe em Ciências da Saúde (Lilacs) e Scientific Eletronic Library Online (SciELO). Foram utilizados Descritores em Ciências da Saúde (DeCS), em português e inglês, combinados pelo operador booleano and. Na busca avançada pelo PubMed, o descritor "minorias sexuais e de gênero" foi combinado com "acesso aos serviços de saúde", "assistência à saúde" e "qualidade da assistência à saúde", separadamente. As mesmas combinações foram realizadas no Lilacs e no SciELO, resultando em pequeno número de artigos. $\mathrm{Na}$ tentativa de ampliar a busca, nessas duas bases, utilizamos também os termos "homossexualidade", "transexualidade" e "pessoas transgêneros", isoladamente, e novos artigos foram compilados.

Delimitaram-se como critérios de inclusão: artigos originais, disponíveis eletronicamente na íntegra, em português, inglês ou espanhol, e publicados entre janeiro de 2007 e dezembro de 2018. Os critérios de exclusão foram: editoriais, cartas ao editor, dissertações e teses, artigos com 
enfoque exclusivamente clínico-epidemiológico, artigos de revisões, ensaios e relatos de experiência. No final de 2006 foram lançados os Princípios de Yogyakarta ${ }^{10}$, documento fundamental para a luta contra o preconceito e discriminação das populações LGBTT no mundo. Com isso, a data de início considerada nessa busca foi 2007.

O primeiro levantamento resultou em 3.428 artigos. Após leitura dos títulos e/ou resumos foram eliminados 448 estudos por serem repetidos e 2.916 pelos critérios de exclusão. Restaram 64 publicações que foram lidas na íntegra. Destas, 23 foram excluídas por não discutiram questões relacionadas ao acesso ou à qualidade da assistência à saúde das populações LGBTT. Ao final, 41 artigos foram incluídos no estudo e identificados por um código alfanumérico. Na Figura 1 , apresentamos esquematicamente as estratégias de busca de acordo com as especificidades de cada base.
Depois de algumas leituras, realizou-se, primeiro, uma análise mais descritiva do material encontrado. Algumas dessas informações foram sintetizadas em um quadro sinóptico (Quadro 1) contendo os seguintes itens: código acompanhado do número de referência, título, primeiro autor, periódico e ano. Em seguida, realizamos uma análise de conteúdo guiada pela problemática da revisão integrativa, na qual as categorias acesso e assistência à saúde das populações LGBTT foram discutidas em três dimensões: relacional, que diz respeito às relações intersubjetivas entre usuários e profissionais; organizacional, que se refere aos modos de organizar os serviços e os processos de trabalho; e contextual, que engloba como situações de vulnerabilidades imbricadas com os determinantes sociais afetam as condições de satisfação das necessidades de saúde.

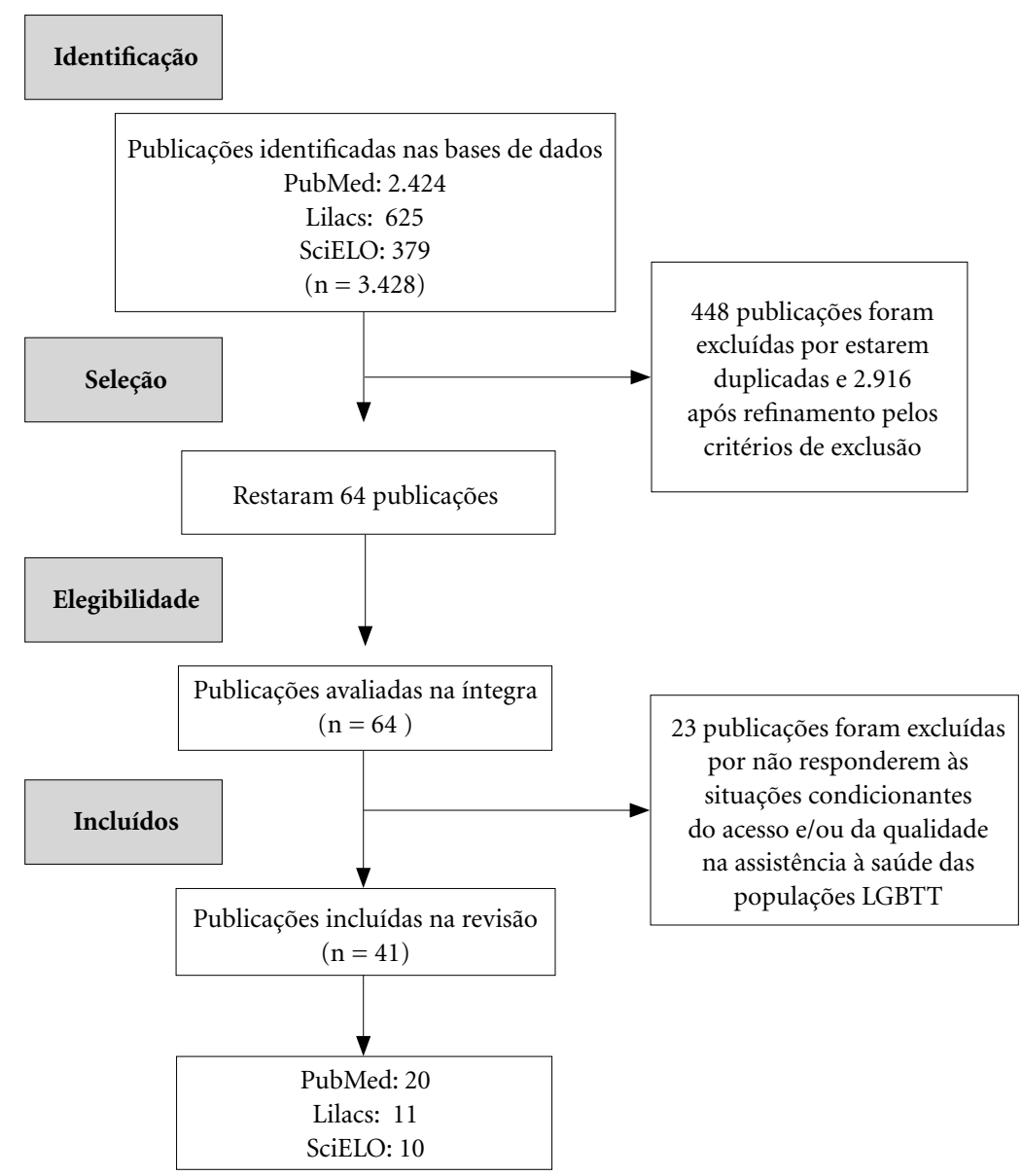

Figura 1. Fluxo do processo de seleção dos artigos para a revisão integrativa. 
Quadro 1. Quadro sinóptico dos artigos selecionados.

\begin{tabular}{|c|c|c|c|c|}
\hline $\begin{array}{l}\text { Código/ } \\
\text { Ref. }\end{array}$ & Título & $\begin{array}{l}\text { Primeiro } \\
\text { Autor }\end{array}$ & Periódico & Ano \\
\hline $\begin{array}{l}\text { A01 } \\
(30)\end{array}$ & $\begin{array}{l}\text { Acesso a cuidados relativos à saúde sexual entre } \\
\text { mulheres que fazem sexo com mulheres em São Paulo, } \\
\text { Brasil }\end{array}$ & Barbosa RM & $\begin{array}{l}\text { Cadernos de Saúde } \\
\text { Pública }\end{array}$ & 2009 \\
\hline $\begin{array}{l}\text { A02 } \\
(14)\end{array}$ & $\begin{array}{l}\text { As experiências dos cuidados de saúde de pessoas } \\
\text { transexuais em Portugal: perspectivas de profissionais } \\
\text { de saúde e utentes }\end{array}$ & Pinto N & Revista de Psicologia & 2012 \\
\hline $\begin{array}{l}\text { A03 } \\
(26)\end{array}$ & $\begin{array}{l}\text { Estudo descritivo da homofobia e vulnerabilidade ao } \\
\text { HIV/Aids das travestis da Região Metropolitana do } \\
\text { Recife }\end{array}$ & Sousa OS & $\begin{array}{l}\text { Ciência \& Saúde } \\
\text { Coletiva }\end{array}$ & 2013 \\
\hline $\begin{array}{l}\text { A04 } \\
(15)\end{array}$ & $\begin{array}{l}\text { Processo de formação das representações sociais sobre } \\
\text { transexualidade dos profissionais de saúde: possíveis } \\
\text { caminhos para superação do preconceito }\end{array}$ & Santos $\mathrm{AB}$ & $\begin{array}{l}\text { Ciência \& Saúde } \\
\text { Coletiva }\end{array}$ & 2014 \\
\hline $\begin{array}{l}\text { A05 } \\
(50)\end{array}$ & $\begin{array}{l}\text { Estigma y discriminación a profesional de la salud } \\
\text { transgénero }\end{array}$ & $\begin{array}{l}\text { Campo- } \\
\text { Arias A }\end{array}$ & $\begin{array}{l}\text { Revista de la } \\
\text { Facultad de Medicina }\end{array}$ & 2014 \\
\hline $\begin{array}{l}\text { A06 } \\
(18)\end{array}$ & $\begin{array}{l}\text { Experiences of homosexual patients' access to primary } \\
\text { health care services in Umlazi, KwaZulu-Natal }\end{array}$ & Cele NH & Curationis & 2015 \\
\hline $\begin{array}{l}\text { A07 } \\
(38)\end{array}$ & $\begin{array}{l}\text { Violência e sofrimento social no itinerário de travestis } \\
\text { de Santa Maria, Rio Grande do Sul, Brasil }\end{array}$ & Souza MHT & $\begin{array}{l}\text { Cadernos de Saúde } \\
\text { Pública }\end{array}$ & 2015 \\
\hline $\begin{array}{l}\text { A08 } \\
(34)\end{array}$ & $\begin{array}{l}\text { Stigma, sexual health, and human rights among women } \\
\text { who have sex with women in Lesotho }\end{array}$ & Poteat TC & $\begin{array}{l}\text { Reproductive Health } \\
\text { Matters }\end{array}$ & 2015 \\
\hline $\begin{array}{l}\text { A09 } \\
(41)\end{array}$ & $\begin{array}{l}\text { Experiências homossexuais de adolescentes: } \\
\text { considerações para o atendimento em saúde }\end{array}$ & Taquette SR & $\begin{array}{l}\text { Interface - } \\
\text { Comunicação, } \\
\text { Saúde, Educação }\end{array}$ & 2015 \\
\hline $\begin{array}{l}\text { A10 } \\
(49)\end{array}$ & $\begin{array}{l}\text { That would have been beneficial: LGBTQ education for } \\
\text { home-care service providers }\end{array}$ & Daley A & $\begin{array}{l}\text { Health \& Social Care } \\
\text { in the Community }\end{array}$ & 2015 \\
\hline $\begin{array}{l}\text { A11 } \\
(27)\end{array}$ & $\begin{array}{l}\text { Outness, stigma, and primary health care utilization } \\
\text { among rural LGBT populations }\end{array}$ & Whitehead J & PLoS One & 2016 \\
\hline $\begin{array}{l}\text { A12 } \\
(28)\end{array}$ & $\begin{array}{l}\text { Dificuldades vividas por pessoas trans no acesso ao } \\
\text { Sistema Único de Saúde }\end{array}$ & Rocon PC & $\begin{array}{l}\text { Ciência \& Saúde } \\
\text { Coletiva }\end{array}$ & 2016 \\
\hline $\begin{array}{l}\text { A13 } \\
(47)\end{array}$ & $\begin{array}{l}\text { Perceived health concerns among sexual minority women } \\
\text { in Mumbai, India: an exploratory qualitative study }\end{array}$ & Bowling J & $\begin{array}{l}\text { Culture, Health \& } \\
\text { Sexuality }\end{array}$ & 2016 \\
\hline $\begin{array}{l}\text { A14 } \\
(46)\end{array}$ & $\begin{array}{l}\text { Health care provision in Brazil: A dialogue between health } \\
\text { professionals and lesbian, gay, bisexual and transgender } \\
\text { service users }\end{array}$ & $\begin{array}{l}\text { Moscheta } \\
\text { MS }\end{array}$ & $\begin{array}{l}\text { Journal of Health } \\
\text { Psychology }\end{array}$ & 2016 \\
\hline $\begin{array}{l}\text { A15 } \\
(31)\end{array}$ & $\begin{array}{l}\text { Lesbian womens' access to healthcare, experiences with } \\
\text { and expectations towards GPs in German primary care }\end{array}$ & Hirsch O & $\begin{array}{l}\text { BMC Family } \\
\text { Practice }\end{array}$ & 2016 \\
\hline $\begin{array}{l}\text { A16 } \\
(40)\end{array}$ & $\begin{array}{l}\text { Barreras en la atención de la salud sexual en Argentina: } \\
\text { percepción de las mujeres que tienen sexo con mujeres }\end{array}$ & Silberman P & $\begin{array}{l}\text { Revista de Salud } \\
\text { Pública }\end{array}$ & 2016 \\
\hline $\begin{array}{l}\text { A17 } \\
(33)\end{array}$ & $\begin{array}{l}\text { Lesbian and bisexual women's sexual healthcare } \\
\text { experiences }\end{array}$ & Munson S & $\begin{array}{l}\text { Journal of Clinical } \\
\text { Nursing }\end{array}$ & 2016 \\
\hline $\begin{array}{l}\text { A18 } \\
(42)\end{array}$ & $\begin{array}{l}\text { 'Competent persons who can treat you with competence, } \\
\text { as simple as that' - an interview study with transgender } \\
\text { people on their experiences of meeting health care } \\
\text { professionals }\end{array}$ & Lindroth M & $\begin{array}{l}\text { Journal of Clinical } \\
\text { Nursing }\end{array}$ & 2016 \\
\hline $\begin{array}{l}\text { A19 } \\
(48)\end{array}$ & $\begin{array}{l}\text { Sentidos atribuídos aos cuidados de saúde e à prevenção } \\
\text { de DST/Aids em específico por jovens gays }\end{array}$ & Cunha RBB & $\begin{array}{l}\text { Physis: Revista de } \\
\text { Saúde Coletiva }\end{array}$ & 2016 \\
\hline $\begin{array}{l}\text { A20 } \\
(51)\end{array}$ & $\begin{array}{l}\text { Da (im)possibilidade do diálogo: conversações públicas } \\
\text { e os direitos LGBTs }\end{array}$ & $\begin{array}{l}\text { Moscheta } \\
\text { MS }\end{array}$ & $\begin{array}{l}\text { Psicologia \& } \\
\text { Sociedade }\end{array}$ & 2016 \\
\hline $\begin{array}{l}\text { A21 } \\
(35)\end{array}$ & $\begin{array}{l}\text { Obstáculos y facilitadores para garantizar el derecho a la } \\
\text { salud integral trans en el Gran Buenos Aires y La Plata }\end{array}$ & Neer AF & $\begin{array}{l}\text { Revista } \\
\text { Argentina de Salud } \\
\text { Pública }\end{array}$ & 2016 \\
\hline $\begin{array}{l}\text { A22 } \\
(22)\end{array}$ & $\begin{array}{l}\text { Unidades Básicas de Saúde em Teresina-PI e o acesso da } \\
\text { população LGB: o que pensam os médicos? }\end{array}$ & Pereira EO & $\begin{array}{l}\text { Tempus Actas de } \\
\text { Saúde Coletiva }\end{array}$ & 2017 \\
\hline
\end{tabular}


Quadro 1. Quadro sinóptico dos artigos selecionados.

\begin{tabular}{|c|c|c|c|c|}
\hline $\begin{array}{l}\text { Código/ } \\
\text { Ref. }\end{array}$ & Título & $\begin{array}{l}\text { Primeiro } \\
\text { Autor }\end{array}$ & Periódico & Ano \\
\hline $\begin{array}{l}\text { A23 } \\
(25)\end{array}$ & $\begin{array}{l}\text { Assistência à saúde da população LGBT em uma capital } \\
\text { brasileira: o que dizem os Agentes Comunitários de } \\
\text { Saúde? }\end{array}$ & $\begin{array}{l}\text { Guimarães } \\
\text { RCP }\end{array}$ & $\begin{array}{l}\text { Tempus Actas de } \\
\text { Saúde Coletiva }\end{array}$ & 2017 \\
\hline $\begin{array}{l}\text { A24 } \\
(37)\end{array}$ & Vivências de travestis no acesso ao SUS & Ferreira BO & $\begin{array}{l}\text { Physis: Revista de } \\
\text { Saúde Coletiva }\end{array}$ & 2017 \\
\hline $\begin{array}{l}\text { A25 } \\
(36)\end{array}$ & $\begin{array}{l}\text { Contemporalis homo sacer: obstáculos para acceder a los } \\
\text { servicios de salud para las poblaciones trans }\end{array}$ & $\begin{array}{l}\text { Caravaca- } \\
\text { Morera JA }\end{array}$ & $\begin{array}{l}\text { Texto \& Contexto - } \\
\text { Enfermagem }\end{array}$ & 2017 \\
\hline $\begin{array}{l}\text { A26 } \\
(20)\end{array}$ & $\begin{array}{l}\text { What lesbian, gay, bisexual, transgender, queer, and } \\
\text { intersex patients say doctors should know and do: a } \\
\text { qualitative study }\end{array}$ & Alpert AB & $\begin{array}{l}\text { Journal of } \\
\text { Homosexuality }\end{array}$ & 2017 \\
\hline $\begin{array}{l}\text { A27 } \\
(21)\end{array}$ & $\begin{array}{l}\text { Health care needs and care utilization among lesbian, gay, } \\
\text { bisexual, and transgender populations in New Jersey }\end{array}$ & Qureshi RI & $\begin{array}{l}\text { Journal of } \\
\text { Homosexuality }\end{array}$ & 2017 \\
\hline $\begin{array}{l}\text { A28 } \\
(43)\end{array}$ & $\begin{array}{l}\text { Scrambling for access: availability, accessibility, } \\
\text { acceptability and quality of healthcare for lesbian, gay, } \\
\text { bisexual and transgender people in South Africa }\end{array}$ & Müller A & $\begin{array}{l}\text { BMC International } \\
\text { Health and Human } \\
\text { Rights. }\end{array}$ & 2017 \\
\hline $\begin{array}{l}\text { A29 } \\
(17)\end{array}$ & $\begin{array}{l}\text { A qualitative study examining young adults' experiences } \\
\text { of disclosure and nondisclosure of LGBTQ identity to } \\
\text { health care providers }\end{array}$ & Rossman K & $\begin{array}{l}\text { Journal of } \\
\text { Homosexuality }\end{array}$ & 2017 \\
\hline $\begin{array}{l}\text { A30 } \\
(13)\end{array}$ & $\begin{array}{l}\text { Health care availability, quality, and unmet need: a } \\
\text { comparison of transgender and cisgender residents of } \\
\text { Ontario, Canada }\end{array}$ & Giblon R & $\begin{array}{l}\text { BMC Health Services } \\
\text { Research }\end{array}$ & 2017 \\
\hline $\begin{array}{l}\text { A31 } \\
(44)\end{array}$ & LGBTQ Youth's Perceptions of Primary Care & Snyder BK & Clinical Pediatrics & 2017 \\
\hline $\begin{array}{l}\text { A32 } \\
(51)\end{array}$ & $\begin{array}{l}\text { The importance of health(ism): A focus group study of } \\
\text { lesbian, gay, bisexual, pansexual, queer and transgender } \\
\text { individuals' understandings of health }\end{array}$ & Graham K & $\begin{array}{l}\text { Journal of Health } \\
\text { Psychology }\end{array}$ & 2017 \\
\hline $\begin{array}{l}\text { A33 } \\
(11)\end{array}$ & $\begin{array}{l}\text { Health for All? Sexual Orientation, Gender Identity, and } \\
\text { the Implementation of the Right to Access to Health Care } \\
\text { in South Africa }\end{array}$ & Müller A & Medical Education & 2017 \\
\hline $\begin{array}{l}\text { A34 } \\
(12)\end{array}$ & $\begin{array}{l}\text { A Política Nacional de Saúde Integral de Lésbicas, } \\
\text { Gays, Bissexuais e Transgêneros (LGBT) e o acesso ao } \\
\text { Processo Transexualizador no Sistema Único de Saúde } \\
\text { (SUS): avanços e desafios }\end{array}$ & $\begin{array}{l}\text { Popadiuk } \\
\text { GS }\end{array}$ & $\begin{array}{l}\text { Ciência \& Saúde } \\
\text { Coletiva }\end{array}$ & 2017 \\
\hline $\begin{array}{l}\text { A35 } \\
(39)\end{array}$ & $\begin{array}{l}\text { O que esperam pessoas trans do Sistema Único de } \\
\text { Saúde? }\end{array}$ & Rocon PC & $\begin{array}{l}\text { Interface - } \\
\text { Comunicação, } \\
\text { Saúde, Educação }\end{array}$ & 2017 \\
\hline $\begin{array}{l}\text { A36 } \\
(29)\end{array}$ & $\begin{array}{l}\text { Disclosure of sexual orientation among women who have } \\
\text { sex with women during gynecological care: a qualitative } \\
\text { study in Brazil. }\end{array}$ & Rufino AC & $\begin{array}{l}\text { The Journal of } \\
\text { Sexual Medicine }\end{array}$ & 2018 \\
\hline $\begin{array}{l}\text { A37 } \\
(32)\end{array}$ & $\begin{array}{l}\text { Práticas sexuais e cuidados em saúde de mulheres que } \\
\text { fazem sexo com mulheres: 2013-2014 }\end{array}$ & Rufino AC & $\begin{array}{l}\text { Epidemiologia e } \\
\text { Serviços de Saúde }\end{array}$ & 2018 \\
\hline $\begin{array}{l}\text { A38 } \\
(16)\end{array}$ & $\begin{array}{l}\text { "Like finding a unicorn": healthcare preferences among } \\
\text { lesbian, gay, and bisexual people in the United States }\end{array}$ & Martos AJ & $\begin{array}{l}\text { Social Science \& } \\
\text { Medicine }\end{array}$ & 2018 \\
\hline $\begin{array}{l}\text { A39 } \\
(24)\end{array}$ & $\begin{array}{l}\text { Diversidade de gênero e acesso ao Sistema Único de } \\
\text { Saúde }\end{array}$ & Ferreira BO & $\begin{array}{l}\text { Revista Brasileira de } \\
\text { Promoção da Saúde }\end{array}$ & 2018 \\
\hline $\begin{array}{l}\text { A40 } \\
(23)\end{array}$ & $\begin{array}{l}\text { Acesso de lésbicas, gays, bissexuais e travestis/ } \\
\text { transexuais às Unidades Básicas de Saúde da Família }\end{array}$ & Oliveira GS & Revista Rene & 2018 \\
\hline $\begin{array}{l}\text { A41 } \\
(19)\end{array}$ & $\begin{array}{l}\text { The no-go zone: a qualitative study of access to sexual } \\
\text { and reproductive health services for sexual and gender } \\
\text { minority adolescents in Southern Africa }\end{array}$ & Müller A & Reproductive Health & 2018 \\
\hline
\end{tabular}




\section{Resultados e discussão}

\section{O que encontramos nos armários?}

A problemática do acesso e da qualidade da assistência à saúde das populações LGBTT tem sido alvo de interesse crescente na literatura nacional e internacional. Entre 2009 e 2014, a produção sobre a temática foi de cinco publicações; e entre 2015 e 2018 identificamos 36 publicações. Em relação ao idioma, 21 artigos eram em língua inglesa, dezessete em português e três em língua espanhola. Cerca da metade dos trabalhos encontrados eram do PubMed, e os demais do Lilacs e SciELO. Os estudos foram desenvolvidos no Brasil (16), Estados Unidos (7), África do Sul (5), Canadá (4), Nova Zelândia (2), Argentina (2), Portugal (1), Suécia (1), Colômbia (1), Índia (1) e Alemanha (1).

Os 41 artigos foram publicados em 30 revistas, sendo dezesseis delas estadunidenses, dez brasileiras, duas colombianas, uma argentina e uma portuguesa. Entre as revistas brasileiras, a Ciência \& Saúde Coletiva teve maior frequência de artigos (4), seguida da Physis (2), Interface (2), Cadernos de Saúde Pública (2), Tempus Actas (2) e as demais apareceram com uma única publicação. No Brasil, essa produção científica tem encontrado espaço em periódicos indexados, de circulação nacional e que se destacam na difusão de estudos de ciências sociais e humanas em saúde. Quase todas as publicações nacionais eram de periódicos classificados como A2, B1 ou B2, na área de saúde coletiva, pelo sistema de avaliação de periódicos da Coordenação de Aperfeiçoamento de Pessoal de Nível Superior (Qualis/ CAPES). A maioria dos periódicos estrangeiros tinha classificação A1, A2 e B2, para a mesma área de conhecimento.

A formação profissional dos primeiros autores dos artigos variou, principalmente, entre Psicologia (12), Enfermagem (10) e Medicina (9). Quatro artigos possuíam um único autor, e um grande contingente três autores ou mais, expressando forte interação entre diferentes centros de pesquisa e diálogos interdisciplinares.

Vinte e nove artigos utilizaram abordagem qualitativa, nove quantitativos e três estudos quanti-qualitativos. Revisões anteriores ${ }^{4,5}$ também apontaram o predomínio da abordagem qualitativa em estudos sobre saúde LGBTT, talvez por serem metodologias mais propícias à compreensão de temas complexos, como as relações de gênero, sexualidade, produção de subjetividade e direitos humanos no campo da saúde. No processo interpretativo, muitos utilizaram técnicas de análise de conteúdo ou análises baseadas em pressupostos hermenêutico-dialéticos. Nas publicações quantitativas, predominaram estudos descritivos com informações sociodemográficas e clínicas de populações LGBTT.

Quanto aos participantes, as pesquisas incluem tanto os grupos LGBTT, como profissionais de saúde, especialmente médicos. Em sua maioria, os estudos utilizam o recrutamento de participantes LGBTT por meio de amostragem de conveniência, a partir da técnica conhecida como Bola de Neve. Notou-se também a incorporação de participantes por recrutamento online ou com apoio de informantes-chave dos movimentos sociais LGBTT. A principal vantagem dos métodos que utilizam cadeias de referência é que, em redes sociais complexas, de difícil acesso, como as populações LGBTT, é mais fácil um membro do grupo conhecer outro membro do que os pesquisadores identificá-los.

Dezenove artigos discutem as populações LGBTT como um único grupo, considerando as nuances em suas análises. Doze estudos utilizam apenas as categorias travesti, homem/ mulher transexual ou transgênero e/ou pessoas trans. Eles discutem os condicionantes biomédicos oriundos do processo de transformação corporal, como a utilização de hormônios, a realização de cirurgias plásticas, as aplicações de silicone, até a própria redesignação sexual. Não obstante, essas análises vão além da perspectiva hegemônica e patológica e os autores buscam desvelar os desafios para a promoção de saúde, a garantia legal desses processos, o fortalecimento dos direitos, do reconhecimento e da autonomia desses sujeitos nos serviços de saúde.Oito artigos abordam a qualidade da assistência à saúde de mulheres lésbicas e bissexuais, em serviços públicos e privados. Estudos exclusivamente com gays masculinos foram encontrados somente em duas publicações, uma nacional e outra internacional. Nenhum artigo analisou somente a condição bissexual. O tema esteve escamoteado nos diferentes estudos, sugerindo que a bissexualidade ocupa um lugar precário socialmente, politicamente e cientificamente, sendo ainda percebida como uma sexualidade "duvidosa", o que dificulta a possibilidade de inclusão real desses sujeitos.Revisão de artigos publicados no Reino Unido também percebeu a pouca produção envolvendo pessoas bissexuais ${ }^{5}$. Apesar da importância do tema, questões relacionadas às necessidades de saúde de adolescentes e jovens LGBTT foram discutidas em somente três artigos. Em alguns estudos (5), 
os pesquisadores - gays, lésbicas e um homem trans - explicitaram, no artigo, sua orientação sexual e/ou identidade de gênero.

\section{Os direitos reconhecidos às populações LGBTT estão nas prateleiras dos armários?}

Os direitos humanos nascem como direitos naturais universais, desenvolvem-se como direitos positivos particulares, para finalmente encontrarem sua plena realização como direitos positivos universais ${ }^{8}$. Nos artigos revisados, as reflexões sobre direitos LGBTT reivindicam legitimidade em um conjunto de documentos de caráter normativo, desde a Declaração Universal dos Direitos Humanos de 1948 à Conferência Internacional sobre População e Desenvolvimento no Cairo de 1994. Os Princípios de Yogyakarta de 2007 - documento que sintetiza um conjunto de princípios para a aplicação da legislação internacional de direitos humanos em relação à orientação sexual e identidade de gênero - foram considerados fundamentais para guiar os Estados na adoção de medidas de proteção às populações LGBTT. As análises sobre as condições de acesso e a qualidade da assistência à saúde, na maioria dos casos, foram enquadradas nesses $\operatorname{marcos}^{11-14}$.

A retirada da homossexualidade da rubrica da patologia foi considerada fato importante para o enfrentamento dos abusos, restrições e negações de direitos às populações LGBTT. Contudo, o processo de desconstrução da perspectiva patologizante tem sido longo e não linear. Os artigos $^{12-15}$ mencionaram o papel dos marcos normativos internacionais quando, em meados dos anos 1970, instâncias como a Associação Americana de Psiquiatria e a Associação Americana de Psicologia deixaram de classificar a homossexualidade como doença e, em 1990, a Organização Mundial de Saúde seguiu o mesmo caminho retirando-a da lista de doenças mentais.

A aprovação de leis que coíbem crimes contra a orientação sexual e identidade de gênero como a lei estadunidense Matthew Shepard e a lei de regulamentação do casamento LGBTT no Canadá - são tomadas como exemplos de iniciativas legais que potencializam a efetivação de direitos $^{13,16,17}$. Os artigos discutem o caso da África do Sul que, mesmo cercada por países avessos aos direitos LGBTT, abriu espaço para a consolidação de uma das legislações mais "progressistas" do mundo; sua Constituição foi a primeira a proibir discriminação sexual e de gênero e, hoje, também protege os refugiados LGBTT, por isso concentra um grande número de "asilados sexuais" do con- tinente $^{11,18,19}$. Em Portugal, a Lei de Identidade de Gênero permite e assiste mudanças corporais e nos registros civis desde 2011, sendo referência para a discussão dos direitos LGBTT $^{14}$.

Na discussão de aspectos da assistência à saúde LGBTT, muitos artigos ${ }^{17,20,21}$, principalmente dos Estados Unidos, tomam como referência documentos do tipo protocolos, manuais técnicos, guias de conduta, recomendações e outros. Majoritariamente, eles apontam lacunas entre os modelos teórico-lógicos das políticas e programas e a realidade da prática clínica.

No cenário brasileiro, muitas publicações citam a Constituição Federal de 1988 que estabeleceu direito universal à saúde ${ }^{15,22,23}$, o programa "Brasil sem Homofobia", de 2004, e a I Conferência Nacional de Políticas Públicas e Direitos Humanos LGBT, de $2008^{12,15,24}$. Na discussão sobre despatologização é muito citada a Resolução 001/99 do Conselho Federal de Psicologia, que proíbe os psicólogos de participar ou propor eventos e serviços que se proponham a "tratar" e "curar" pessoas LGBTT ${ }^{15,23}$. Na discussão sobre o processo transexualizador no SUS, o marco é a Portaria no 2.803/13, do Ministério da Saúde, apontado com divisor de águas na perspectiva da equidade em saúde ${ }^{4,25}$. A Portaria ministerial $n^{\circ}$ 2.836/11 que lançou a Política Nacional de Saúde Integral de Lésbicas, Gays, Bissexuais, Travestis e Transexuais é referência para as discussões sobre acesso e qualidade da assistência à saúde dessas populações ${ }^{12,23,24}$.

Estudos mostram como problemas históricos e estruturais dos serviços e dos sistemas de saúde, ligados a contextos políticos, econômicos e culturais, afetam a produção da saúde de indivíduos com múltiplas estigmatizações - por gênero, raça/etnia, situação socioeconômica, dentre outras $^{11,16,26,27}$. Na realidade brasileira, apesar da existência de políticas de saúde para as populações LGBTT, gestores de saúde enfrentam dificuldades na operacionalização, monitoramento e avaliação das mesmas, e há fragilidades na coordenação e no diálogo entre representantes dos governos federal, estadual e municipal ${ }^{12,23}$.

Se a literatura nacional e internacional mostra como, para a garantia de direitos LGBTT, são importantes os instrumentos normativos e legais, revela também que eles não são suficientes para as mudanças. Para sua eficácia concreta, precisam ser estabelecidas pactuações de outras ordens que não normativas e jurídicas e que passam por circuitos que não aqueles macropolíticos e macroinstitucionais. As lutas políticas que passam pelas instâncias que desenham, implementam e 
geram as políticas públicas, são transladadas e reencenadas nos serviços de saúde, nos corredores das unidades, no interior dos consultórios, nos encontros e nas sociabilidades que ali diuturnamente se tecem.

\section{Des-montando os armários do acesso e da qualidade da assistência à saúde LGBTT}

Neste tópico, analisamos as discussões da literatura sobre as condições que influenciam o acesso e a qualidade da assistência à saúde das populações LGBTT. Uma vasta produção teórica nacional e internacional tem investido em categorias e modelos de análise para o estudo de acesso e qualidade em saúde, sob perspectivas heterogêneas, que vão desde estudos avaliativos normativos até aqueles mais sociológicos. Sem pretender aprofundamento em questões mais técnicas da área de avaliação em saúde, nesta revisão, os temas e as discussões encontrados nos artigos foram organizados em três dimensões principais: relacional, que diz respeito às relações intersubjetivas entre usuários e profissionais de saúde; organizacional, que se refere às estratégias de organizar os serviços e os processos de trabalho; e, por fim, contextual, que mostra como diferentes questões de vulnerabilidade afetam as situações de saúde. A distinção entre essas dimensões é meramente para fins de operacionalizar a análise; nas publicações - assim como nos fluxos de interação da vida real - elas são complexas e imbricadas.

\section{A dimensão relacional}

$\mathrm{Na}$ dimensão chamada aqui "relacional", as relações entre pessoas LGBTT e profissionais de saúde foram identificadas como condição central à qualidade da assistência, pois delas dependem o acolhimento, que implica construção de vínculos, respeito, não discriminação e não julgamento, isto é, a prática de uma clínica comprometida com a cidadania ${ }^{25,28}$.

A literatura sobre condições de acesso aos serviços de saúde mostra que a boa relação usuário -profissional pressupõe uma escuta ativa e qualificada ${ }^{1,3}$. Nos artigos revisados, essa qualificação da relação foi apontada como uma condição para a revelação da orientação sexual e/ou identidade de gênero nos serviços de saúde - condição indispensável para uma assistência resolutiva e o estabelecimento de linhas de cuidado ${ }^{22,29}$. As populações LGBTT mostraram preocupações diversas no que se refere a revelar-se ou não aos profissionais de saúde ${ }^{29-31}$.
Pesquisa com mulheres lésbicas em São Paulo mostrou que a principal barreira na busca pelos serviços de saúde está relacionada ao receio de revelar sua orientação sexual e práticas homoeróticas, e à expectativa de sofrer preconceitos ${ }^{30}$. Em estudo realizado na Alemanha com 766 mulheres lésbicas, apenas $40 \%$ revelaram a sua orientação sexual aos profissionais de saúde, embora $89 \%$ delas tivessem um médico de referência para os cuidados básicos $^{31}$. A não revelação pode deixar as mulheres lésbicas mais vulneráveis às infecções sexualmente transmissíveis (IST) e Aids, pois, apesar de suas variadas práticas sexuais, elas pouco utilizam preservativos ${ }^{32}$.

Na África do Sul, as populações LGBTT descreveram experiências de desrespeito por parte de profissionais de saúde ou administrativos, ao revelarem a orientação sexual e/ou identidade de gênero nos serviços de saúde, com cenas de assédio verbal e sexual ${ }^{19,20}$. Ao expressaram o desejo pela gravidez, mulheres lésbicas da Nova Zelândia se sentiram assediadas através de reações pejorativas dos profissionais de saúde ${ }^{33}$. Outro estudo mostrou que usuários que se revelaram tiveram 2,5 vezes mais chances de sofrer preconceitos $^{34}$.

A revelação da orientação sexual e/ou identidade de gênero pode causar constrangimentos nos profissionais de saúde pouco sensibilizados ou capacitados para atender essas populações. Pesquisas ${ }^{29,33}$ mostraram que após a verbalização da identidade LGBTT, usuários perceberam que os profissionais se puseram desconfortáveis, com rápida mudança de assunto, não sabendo como continuar a comunicação, ou então, incrédulos diante da revelação da orientação sexual e/ ou identidade de gênero. Nos atendimentos, alguns usuários também perceberam curiosidade inadequada dos profissionais de saúde, em que perguntas e detalhes das práticas sexuais foram questionadas, sem que essas inquietações fossem relevantes para o resgate histórico, diagnóstico ou tratamento em saúde ${ }^{19,20}$. Eles destacaram também que as crenças religiosas dos profissionais, quando impostas, fragilizavam o vínculo ${ }^{18,22}$.

A violação do sigilo das informações nos atendimentos em saúde LGBTT foi um tema bastante abordado na literatura investigada e em outras revisões ${ }^{4,5}$. Usuários relataram que sua orientação sexual e/ou identidade de gênero foi compartilhada com outros profissionais, de forma indiscriminada e desnecessária, bem como com outras pessoas da comunidade ${ }^{35,36}$. Nos Estados Unidos, os usuários relataram medo de que suas informações fossem passadas para segura- 
doras de saúde e taxas maiores fossem cobradas, associando a identidade LGBTT ao risco de contrair HIV ${ }^{17}$. Pessoas trans canadenses apresentaram dificuldades na adesão aos seguros de saúde, estando em listas separadas com espera de mais de um ano para efetivar o seguro ${ }^{13}$.

\section{A dimensão organizacional}

Os modos de organização dos serviços, os processos de trabalho e os efeitos no que se refere ao acesso e à qualidade da assistência à saúde das populações LGBTT foram problematizados na literatura. A inadequação do acolhimento foi evidenciada em situações diversas como: o não reconhecimento do nome social no prontuário e nas comunicações nos balcões, nas salas de espera e nos consultórios, relatado por travestis brasilei$\operatorname{ras}^{37-39}$, a dispensação rotineira de preservativos masculinos às mulheres lésbicas, sem considerar suas práticas sexuais ${ }^{40}$; a confusão entre a transexualidade e a homossexualidade ${ }^{15}$; a exposição de adolescentes LGBTT a situações vexatórias ou a quebra de privacidade diante os pais ou responsáveis $^{20,41}$.

A lógica organizacional heteronormativa e seus efeitos nos processos de trabalho e comunicação nos serviços de saúde foram percebidas pelos sujeitos participantes dos estudos revisados, bem como foram destacados em outras revisões anteriores $^{4,5}$. Na ambiência dos serviços, especialmente nas salas de espera, usuários destacaram a falta de atividades e recursos informativos e educativos - cartazes, folhetos, cartilhas, palestras referentes à saúde das populações LGBTT $^{12,42-44}$. A criação de um ambiente favorável a essas populações nos serviços requer, entre outras coisas, a promoção institucional de processos pedagógicos que inclua o respeito à diversidade sexual e de gênero e fomente uma cultura de valorização de direitos humanos. Destarte, há importantes fragilidades nas instituições de saúde, no tocante a esse papel educativo ${ }^{23}$. Um artigo mostrou que os materiais educativos utilizados nos serviços tinham uma linguagem muito técnica, pouco LGBTT-friendly, que não se aproximava dos dialetos e gírias próprios das comunidades ${ }^{33}$. Pessoas LGBTT perceberam também que estavam sendo julgadas por trabalhadores de serviços de limpeza, segurança, e administração das instituições de saúde, por conta da sua forma de falar, suas vestimentas ou modos de se expressar ${ }^{24}$. Outros relataram que se sentiam rejeitados pelos demais usuários dos serviços na sala de espera ${ }^{23}$. Em vários artigos ${ }^{18,43}$, os próprios usuários sugeriram a realização de ações educativas para os gestores, administradores, profissionais de saúde e outros que atuam em serviços de apoio, bem como para a comunidade em geral, com o intuito de desmistificar conceitos historicamente construídos - o que poderia ter efeitos positivos tanto na dimensão relacional como na organizacional.

A transformação dos processos de trabalho nas instituições de saúde exige dos profissionais maior sensibilidade e compreensão dos direitos e das especificidades de assistência às populações LGBTT, especialmente para as pessoas trans ${ }^{12}$. Nos Estados Unidos, populações LGBTT da zona rural relatam dificuldades de encontrar provedores de saúde disponíveis e capacitados ${ }^{27}$. Em Portugal, essas populações foram melhor assistidas por profissionais que possuíam familiares LGBTT, indicando uma sensibilidade gerada a partir da própria vivência sistêmica ${ }^{14}$.

Além da sua dimensão pedagógica, a educação permanente - capacitações e treinamentos, as modalidades mais discutidas na literatura - voltada para a saúde LGBTT foi mencionada como importante estratégia de mudança na lógica operacional dos serviços ${ }^{23,44}$. Iniciativas propulsoras desse debate, como a da Association of American Medical Colleges, que tem disponibilizado vídeos, guias e manuais informativos para os profissionais de saúde, foram discutidas ${ }^{17}$. Do mesmo modo, discutiram-se iniciativas do Ministério da Saúde do Brasil que, em parcerias com universidade públicas, tem promovido cursos e formações sobre a temática LGBTT, na modalidade a distância ${ }^{22,25}$. A inclusão da questão dos direitos LGBTT na formação acadêmica foi outro tema evidenciado. Profissionais de saúde entrevistados apontaram fragilidades do ensino ofertado nas faculdades; consideraram que os conteúdos curriculares referentes à saúde LGBTT transmitem uma concepção exclusivamente tecnicista e biomédica, com enfoque nas IST e nos riscos associados $^{12,25,31}$.

Os sentidos atribuídos às necessidades e aos cuidados de saúde das populações LGBTT estão imersos numa rede contínua de enfrentamentos, negociações, legitimações e transformações, que podem aproximar ou afastar esses sujeitos dos serviços, e por isso é necessária uma discussão mais ampla e permanente nos aparelhos formadores e nos processos de educação em serviço.

\section{A dimensão contextual}

Em uma série de estudos, discutem-se situações de vulnerabilidade e determinantes sociais 
que afetam as condições de satisfação das necessidades de saúde das populações LGBTT. As noções de vulnerabilidade e de determinação social em saúde remetem ao entrelaçamento de condições materiais, psicológicas, culturais, morais, jurídicas e políticas que privam as pessoas, em graus variados, de direitos, autonomia, reconhecimento e participação, as expõe a situações de adoecimento e as excluem dos recursos disponíveis à saúde ${ }^{41-48}$. O que é chamado aqui de universo LGBTT é um conglomerado heterogêneo de pessoas, que se distinguem não apenas pelas suas identidades sexuais e/ou de gênero, mas por marcadores de classe, raça/etnia, origem e outros.

As situações de pobreza, violência, discriminação e estigmatização são componentes importantes de contextos que vulnerabilizam e precarizam as condições de saúde de pessoas LGBTT, embora nem sempre do mesmo modo ${ }^{15,26}$. Alguns estudos relevaram que imigrantes, indígenas, pessoas privadas de liberdade, negros e residentes da zona rural sofrem vulnerabilidades ainda mais acentuadas, na tentativa de acesso aos serviços de saúde ${ }^{27,43}$.

Um estudo com as populações LGBTT de New Jersey mostrou que, entre as dificuldades de acesso aos serviços de saúde, está o custo dos seguros particulares ${ }^{21}$. Nas realidades brasileira e sul-africana, as populações LGBTT em situação de pobreza enfrentam fragilidades para acessar serviços e ações de saúde pela dificuldade, por exemplo, de pagar o transporte para chegar às unidades de saúde ${ }^{11,28}$. No caso das pessoas trans, na tentativa de se tornarem inteligíveis, a escassez ou inexistência de oferta de certas tecnologias e procedimentos especializados nos serviço públicos faz com que elas, muitas vezes, se submetam a processos de forja dos seus corpos em serviços clandestinos, sem nenhum tipo de segurança, e inclusive acabam vivenciando experiências de quase morte constantemente ${ }^{37-39}$.

A LGBTTfobia, termo que denota discriminações e as mais variadas formas de violência sofridas pelas populações LGBTT, se prolonga e reproduz na cadeia de eventos que vai dos maustratos e espancamentos dentro das famílias, nas ruas ou no trabalho até nas instituições onde se busca apoio, como as delegacias e serviços de saúde $^{16}$. Pessoas trans, grupo para o qual muitas vezes a busca pelos serviços de saúde está associado a esses eventos, relataram o não uso proposital do nome social, as chacotas e as brincadeiras vexatórias nas salas de emergência ${ }^{12,14,19}$. A violência institucional gera desconfiança dos profissionais, obstaculiza os vínculos e produz a atitude de evitar os serviços de saúde em outras situações ${ }^{38}$.
Outro aspecto discutido é como a discriminação e a violência, além de serem condicionantes do acesso e da qualidade da assistência à saúde, são também fatores de adoecimentos. Essas situações são associadas com ansiedade, depressão, tentativas de suicídio, uso abusivo de substâncias psicoativas, e precisam ser consideradas pelos serviços de saúde ${ }^{17,47}$.

Com a epidemia de Aids, a homossexualidade e outras sexualidades e gêneros divergentes da norma ficaram atreladas, de forma perversa, ao adoecimento, transformando a própria identidade sexual em sinônimo de Aids. Alguns artigos discutiram como o duplo estigma - o da transgressão da heteronormatividade e o da Aids -, em seus vários atravessamentos, também traspassavam os serviços de saúde na América Latina ${ }^{48}$, no Canadá ${ }^{49}$ e nos Estados Unidos ${ }^{25}$, operando tanto na assistência básica como na especializada. $\mathrm{Na}$ Índia, com o aumento de HIV em pessoas trans, essa dupla estigmatização foi ainda mais reforça$\mathrm{da}^{47}$. Um estudo argentino mostrou outro modo de estigma: pessoas trans profissionais de saúde foram avaliadas pelos usuários como infectantes de HIV, pedófilas ou criminosas sexuais, antiéticas ou pouco competentes em sua profissão $0^{50}$.

O reconhecimento e a proteção dos direitos humanos são os pilares de sustentação de uma democracia $^{8}$. Nas publicações revisadas, discutiu-se a importância dos ativismos LGBTT nas lutas pelos direitos humanos no campo da saúde e, por conseguinte, nas lutas por transformar, em um sentido positivo, as próprias condições contextuais e intrínsecas aos serviços e às práticas profissionais que influenciam o acesso e a qualidade da assistência à saúde LGBTT ${ }^{45,46,51}$.

\section{Considerações finais}

O imaginário instituinte da sociedade não é mera imagem mental de alguma coisa, é criação incessante "social-histórica e psíquica" de formas de se relacionar, agir e pensar que produzem realidades e racionalidades ${ }^{7}$. A literatura nacional e internacional nos indica como o imaginário instituinte da heteronormatividade tem perpassado, especialmente, as práticas nos serviços de saúde - seja nas salas de espera ou no interior dos consultórios - e afetado as condições de acesso e qualidade da assistência à saúde das populações LGBTT. As pessoas cuja identidade de gênero e orientação sexual são distintas do que propõe o binarismo sexual são alvo de preconceitos, violências e discriminações, que somadas aos marcadores de classe, 
raça/etnia e origem engendram um contexto de vulnerabilidades no campo da saúde.

Os avanços das leis, normas, políticas e programas de direitos humanos, especialmente os referentes à saúde das populações LGBTT, são reconhecidos. Porém, para além desses importantes marcos normativos, há a necessidade de se pensar estratégias de transformação de práticas e interações face-a-face que se dão nos interstícios e no cotidiano dos serviços de saúde. Do contrário, corre-se o risco de se afastar cada vez mais parcelas das populações LGBTT dessas instituições. As normativas legais só se realizam quando traduzidas às dinâmicas locais, ou seja, incorporadas e compartilhadas por gestores, profissionais e usuários dos serviços.

É necessário interligar, dentro de um mesmo princípio de justiça, o espaço de reconhecimento das injustiças de gênero (campo social e cultural) e o espaço das desigualdades atreladas à exploração e redistribuição de recursos (campo econômico $)^{52}$. Essa perspectiva impõe a reflexão que as barreiras enfrentadas pelas populações LGBTT nos serviços de saúde são produto da desvalorização ou falta de reconhecimento de suas identidades que, numa lógica circular, produzem ou acentuam restrições no que tange ao acesso a bens e recursos sociais, inclusive os cuidados em saúde.

Revelar-se LGBTT ou não nas interações, como os serviços de saúde, despontou na literatura como questão de muita relevância nos processos de produção de saúde dessas populações. Aqui, poderíamos perguntar sobre o "lugar de fala"53 dos dissidentes sexo-gênero e as hierarquias de poder instituídas no campo da saúde. A palavra lugar vem bem a calhar. A deduzir da literatura, os serviços de saúde têm sido extensões - gavetas e prateleiras - daquela invisibilidade e negação que tem sido referida com a metáfora do armário. Portanto, revelar-se é parte iniludível do rompimento com essa lógica, é operação necessária para que se possam abrir os armários e as populações LGBTT possam ter seus direitos reconhecidos, acessados e realizados.

\section{Colaboradores}

BO Ferreira e C Bonan contribuíram igualmente em todas as etapas de construção do manuscrito. 


\section{Referências}

1. Giovanella L, Fleury S. Universalidade da Atenção à Saúde: acesso como categoria de análise. In: Eibenschutz C, organizadora. Política de Saúde: o público e o privado. Rio de Janeiro: Fiocruz; 1995. p. 177-198.

2. Donabedian A. Aspects of medical care administration: specifying requirements for health care. Cambridge: Harvard University; 1973.

3. Uchimura KY, Bosi MLM. Qualidade e subjetividade na avaliação de programas e serviços em saúde. Cad Saude Publica 2002; 18(6):1561-1569.

4. Albuquerque GA, Garcia CL, Quirino GS, Alves MJA, Belém JM, Figueiredo FWS, Paiva LS, Nascimento VB, Maciel ES, Valenti VE, Abreu LC, Adami F. Access to health services by lesbian, gay, bisexual, and transgender persons: systematic literature review. BMC Int Health Hum Rights 2016;16(2):1-10.

5. Meads C, Carmona C, Kelly MP. Lesbian, gay and bisexual people's health in the UK: a theoretical critique and systematic review. Diversity and Equality in Health and Care 2012; 9(6):19-32.

6. Bento B. A reinvenção do corpo: sexualidade e gênero na experiência transexual. Rio de Janeiro: Garamond; 2006.

7. Castoriadis C. A instituição imaginária da sociedade. Rio de Janeiro: Paz e Terra; 1982.

8. Bobbio N. A era dos direitos. Rio de Janeiro: Campus; 2004.

9. Mendes KDS, Silveira RCCP, Galvão CM. Revisão integrativa: método de pesquisa para a incorporação de evidências na saúde e na enfermagem. Texto contexto - enferm 2008; 17(4):758-764.

10. Corrêa SO, Muntarbhorn V, organizadores. Princípios de Yogyakarta: princípios sobre a aplicação da legislação internacional de direitos humanos em relação à orientação sexual e identidade de gênero. Rio de Janeiro: Observatório de Sexualidade e Política: 2006.

11. Müller A. Health for All? Sexual Orientation, Gender Identity, and the Implementation of the Right to Access to Health Care in South Africa. Health Hum Rights 2016; 18(2):195-208.

12. Popadiuk GS, Oliveira DC, Signorelli MC. A Política Nacional de Saúde Integral de Lésbicas, Gays, Bissexuais e Transgêneros (LGBT) e o acesso ao Processo Transexualizador no Sistema Único de Saúde (SUS):avanços e desafios. Cien Saude Colet 2017; 22(5):1509-1520.

13. Giblon R, Bauer GR. Health care availability, quality, and unmet need: a comparison of transgender and cisgender residents of Ontario, Canada. BMC Health Serv Res 2017; 17(1):283-292.

14. Pinto N, Moleiro C. As experiências dos cuidados de saúde de pessoas transexuais em Portugal: perspectivas de profissionais de saúde e utentes. Psicologia 2012; 26(1):129-151.

15. Santos AB, Shimizu HE, Merchan-Hamann E. Processo de formação das representações sociais sobre transexualidade dos profissionais de saúde: possíveis caminhos para superação do preconceito. Cien Saude Colet 2014; 19(11):4545-4554.
16. Martos AJ, Wilson PA, Gordon AR, Lightfoot M, Meyer IH. "Like finding a unicorn": Healthcare preferences among lesbian, gay, and bisexual people in the United States. Soc Sci Med 2018; 209(7):126-133.

17. Rossman K, Salamanca P, Macapagal K. A Qualitative Study Examining Young Adults' Experiences of Disclosure and Nondisclosure of LGBTQ Identity to Health Care Providers. J Homosex 2017; 64(10):13901410.

18. Cele NH, Sibiya MN, Sokhela DG. Experiences of homosexual patients' access to primary health care services in Umlazi, KwaZulu-Natal. Curationis 2015; 38(2):1522-1530.

19. Müller A, Spencer S, Meer T, Daskilewicz K. The nogo zone: a qualitative study of access to sexual and reproductive health services for sexual and gender minority adolescents in Southern Africa. Reprod Health 2018; 15(1):12-21.

20. Alpert AB, CichoskiKelly EE, Fox AD. What lesbian, gay, bisexual, transgender, queer, and intersex patients say doctors should know and do: A qualitative study. $J$ Homosex 2017; 64(3):1368-1389.

21. Qureshi RI, Zha P, Kim S, Hindin P, Naqvi Z, Holly C, Dubbs W, Ritch W. Health care needs and care utilization among lesbian, gay, bisexual, and transgender populations in New Jersey. J Homosex 2018; 65(2):167-180.

22. Pereira EO, Ferreira BO, Amaral GS, Cardoso CV, Lorenzo CFG. Unidades Básicas de Saúde em Teresina-PI e o acesso da população LGBT: o que pensam os médicos? Tempus, Actas de Saúde Colet 2017; 11(1):51-67.

23. Oliveira GS, Nogueira JA, Costa GPO, Silva FVS, Almeida AS. Acesso de lésbicas, gays, bissexuais e travestis/transexuais às Unidades Básicas de Saúde da Família. Revista Rene 2018; 19(1):1-8.

24. Ferreira BO, Pedrosa JIS, Nascimento EF. Diversidade de Gênero e Acesso ao Sistema Único de Saúde. Rev. Bras. Proç. da Saúde 2017; 31(1):1-10.

25. Guimarães RCP, Cavadinha ET, Mendonça AVM, Sousa MF. Assistência à saúde da população LGBT em uma capital brasileira: o que dizem os Agentes Comunitários de Saúde? Tempus, Actas de Saúde Colet 2017; 11(1):121-139.

26. Sousa P, Ferreira LO, Sá J. Estudo descritivo da homofobia e vulnerabilidade ao HIV/Aids das travestis da Região Metropolitana do Recife. Cien Saude Colet 2013; 18(8):2239-2251.

27. Whitehead J, Shaver J, Stephenson R. Outness, Stigma, and Primary Health Care Utilization among Rural LGBT Populations. PLoS ONE 2016; 11(1):e0146139.

28. Rocon PC, Rodrigues A, Zamboni J, Pedrini MD. Dificuldades vividas por pessoas trans no acesso ao Sistema Único de Saúde. Cien Saude Colet 2016; 21(8):2517-2526.

29. Rufino AC, Madeiro A, Trinidad AS, Rodrigues SR, Freitas I. Disclosure of Sexual Orientation Among Women Who Have Sex With Women During Gynecological Care: A Qualitative Study In Brazil. J Sex Med 2018; 15(7):966-973. 
30. Barbosa RM, Facchini R. Acesso a cuidados relativos à saúde sexual entre mulheres que fazem sexo com mulheres em São Paulo, Brasil. Cad Saude Publica 2009; 25(Supl. 2):s291-s300-308.

31. Hirsch O, Löltgen K, Becker A. Lesbian womens' access to healthcare, experiences with and expectations towards GPs in German primary care. BMC Fam Pract 2016; 17(1):162-169.

32. Rufino AC, Madeiro A, Trinidad AS, Rodrigues SR, Freitas I. Práticas sexuais e cuidados em saúde de mulheres que fazem sexo com mulheres: 2013-2014. Epidemiologia e Serviços de Saúde 2018; 27(4):e2017499.

33. Munson S, Cook C. Lesbian and bisexual women's sexual healthcare experiences. J Clin Nurs 2016; 25(3):3497-3510.

34. Poteat TC, Logie CH, Adams D, et al. Stigma, sexual health, and human rights among women who have sex with women in Lesotho. Reprod Health Matters 2015; 23(46):107-116.

35. Neer AF. Obstáculos y facilitadores para garantizar el derecho a la salud integral trans en el Gran Buenos Aires y La Plata. Rev. Argent. Salud Publica 2016; 7(29):26-30.

36. Caravaca-Morera JA, Bennington M, Wiliams C, Mackinnon K, Ross LE. Contemporalis homo sacer: obstáculos para acceder a los servicios de salud para las poblaciones trans. Texto \& Contexto - Enfermagem 2017; 26(3):e3710016.

37. Ferreira BO, Nascimento EF, Pedrosa JIS, Monte LMI. Vivências de travestis no acesso ao SUS. Physis 2017; 27(4):1023-1038.

38. Souza MHT, Malvasi P, Signorelli MC, Pereira PPG. Violência e sofrimento social no itinerário de travestis de Santa Maria, Rio Grande do Sul, Brasil. Cad Saude Publica 2015; 31(4):767-776.

39. Rocon PC, Sodré F, Zamboni J, Rodrigues A, Roseiro MCFB. O que esperam pessoas trans do Sistema Único de Saúde? Interface (Botucatu) 2018; 22(6):43-53.

40. Silberman P, Buedo, PE, Burgos, LM. Barreras en la atención de la salud sexual en Argentina: percepción de las mujeres que tienen sexo con mujeres. Rev. Salud Pública 2016; 18(1):1-10.

41. Taquette SR, Rodrigues AO. Experiências homossexuais de adolescentes: considerações para o atendimento em saúde. Interface (Botucatu) 2015; 19(55):11811191.

42. Lindroth M. Competent persons who can treat you with competence, as simple as that - an interview study with transgender people on their experiences of meeting health care professionals. J Clin Nurs 2016; 25(1):3511-3521.

43. Müller A. Scrambling for access: availability, accessibility, acceptability and quality of healthcare for lesbian, gay, bisexual and transgender people in South Africa. BMC Int Health Hum Rights 2017; 17(1):16-21.

44. Snyder BK, Burack GD, Petrova A. LGBTQ youth's perceptions of primary care. Clinical Pediatric 2017; 56(1):443-450.

45. Moscheta M, Souza LV, Casarini KA, Scorsolini-Comin F. Da (im)possibilidade do diálogo: conversações públicas e os direitos LGBTS. Psicologia \& Sociedade 2016; 28(3):516-525.
46. Moscheta MS, Souza LV, Santos, MA. Health care provision in Brazil: A dialogue between health professionals and lesbian, gay, bisexual and transgender service users. J Health Psychol 2016; 21(3):369-378.

47. Bowling J, Dodge B, Banik S, Rodriguez I, Mengele SR, Herbenick D, Guerra-Reyes L, Sanders S, Dange A, Anand V. Perceived health concerns among sexual minority women in Mumbai, India: an exploratory qualitative study. Cult Health Sex 2016; 18(7):826-840.

48. Cunha RBB, Gomes R. Sentidos atribuídos aos cuidados de saúde e à prevenção de DST/Aids em específico por jovens gays. Physis 2016; 26(3):807-828.

49. Daley A, MacDonnell JA. 'That would have been beneficial': LGBTQ education for home-care service providers. Health Soc Care Community 2015; 23(3):282291.

50. Campo-Arias A, Oviedo HC, Herazo, E. Estigma y discriminación a profesional de la salud transgénero. Revista de la Facultad de Medicina 2014; 62(1):41-45.

51. Graham K, Treharne GJ, Ruzibiza C, Nicolson M. The importance of health(ism):A focus group study of lesbian, gay, bisexual, pansexual, queer and transgender individuals' understandings of health. J Health Psychol 2017; 22(2):237-247.

52. Fraser N. Justice Interruptus: Critical Reflections on the "Postsocialist" Condition. New York: Routledge; 1997.

53. Ribeiro D. O que é lugar de fala? Belo Horizonte: Letramento; 2017.
Artigo apresentado em 30/05/2019 Aprovado em 07/08/2019 Versão final apresentada em 27/11/2019 
